\title{
The shape of occupancy distributions in plant communities: the importance of artefactual effects
}

\author{
Peter M. Kammer and Corinne M. Vonlanthen
}

Kammer, P. M. and Vonlanthen, C. M. 2009. The shape of occupancy distributions in plant communities: the importance of artefactual effects. - Web Ecol. 9: 8-23.

\begin{abstract}
Occupancy frequency distributions are commonly used as an approach to describe and analyse interspecific distribution patterns. However, the relative importance of biological versus artefactual mechanisms in shaping occupancy distributions is still largely undetermined. We evaluated the importance of different and interacting artefactual effects on the shape of occupancy distributions in local plant communities. The effects of sampling protocol parameters (i.e. size and number of sample units, sample extent, coverage, and intensity) on the shape of the occupancy distributions were examined separately. We identified the mechanisms that cause the effects by tracking the shifts of individual species between occupancy classes with varying parameters. Furthermore, the impact of different species abundance distributions and increasing levels of intraspecific aggregation on occupancy distributions was investigated by means of artificial assemblages. We show the following results: 1) increases in the number of sample units, sample extent, coverage, and intensity all result in a unimodal occupancy distribution with the mode in the lowest occupancy class; 2) an increase in sample unit size leads to a bimodal distribution; 3) changes that occur in the shape of the occupancy distributions with varying sampling protocol parameters can be explained by the movements of the species between occupancy classes; 4) different species abundance distributions may cause occupancy distributions with a left-hand mode or a bimodal distribution; and 5) the number of species in the highest occupancy class decreases with increasing degree of aggregation. The mode that almost always occurs in the lowest occupancy class is most likely due to the high number of rare species existing in most communities; the mode in the highest class emerges as a pure artefact that occurs when the sample unit size is relatively large compared to the sample extent. Consequently, the exclusion or separation of concurrent artefactual mechanisms is crucial when investigating the biological causes for the shape of occupancy distributions.
\end{abstract}

P. M. Kammer (peter.kammer@phbern.ch), Biology Dept, Univ. of Teacher Education, Gertrud-Wokerstrasse 5, CH-3012 Bern, Switzerland. - C. M. Vonlanthen, Inst. of Geography, Univ. of Bern, Hallerstrasse 12, CH-3012 Bern, Switzerland. Present address: Federal Office for the Environment, CH-3003 Bern, Switzerland.

Explaining species distributions is one of the main focuses of ecology and biogeography. Indeed, in light of global warming, which involves shifts of the ranges and distributions of species (Walther et al. 2002, Parmesan 2006), an improved understanding of distribution patterns and their underlying processes is desirable. Occupancy frequency distributions, henceforth referred to as 'occupancy distributions', are defined as plotting the number of species (i.e. frequency) against the proportion of the total number of sample units in which the species occur (i.e. occupancy).

Accepted 12 February 2009

Copyright (C) EEF

ISSN 1399-1183 
This approach is commonly used to describe and analyse interspecific distribution patterns. In particular, the often observed occurrence of a bimodal occupancy distribution with a peak in the lowest and the highest occupancy class has become an object of attention and was primarily described in insect and plant communities (see compilations in Tokeshi 1992, Scheiner and Rey-Benayas 1997, McGeoch and Gaston 2002). Recent studies have documented occupancy distributions for a broad range of taxonomic groups and spatial systems ranging from epiphytic plants in temperate and tropical forests (Löbel et al. 2006, Laube and Zotz 2007) to freshwater diatoms, insects, and bryophytes in boreal streams (Heino 2005, Soininen and Heino 2005, Heino and Virtanen 2006). Multiple hypotheses have been proposed to explain the different shapes of the observed occupancy distributions. They include equilibrium approaches, such as underlying habitat heterogeneity (Raunkiaer 1934) or the niche-based explanation of Brown (1984), which suggests that the bimodality of an occupancy distribution results from the contrast between generalists that are present in most habitat patches and specialists, which are typically confined to a small number of patches. Furthermore, non-equilibrium mechanisms were also proposed, including the dynamic meta-population model (Hanski 1982a), which proposes that bimodality is a result of the stochastic processes of local colonisation and extinction, thereby predicting that most species will be either frequent within a region (i.e. core species) or rare (i.e. satellite species). This core-satellite dichotomy has repeatedly been used as the theoretical basis for the distinction of species groups with differing biological characteristics (Glenn and Collins 1990, Gibson et al. 1999, Pärtel et al. 2001, Ulrich and Zalewski 2006).

However, from the advent of occupancy distributions, it has been stated that the method of sampling, i.e. the sampling protocol, may have a significant effect on the final shape of the distribution (McGeoch and Gaston 2002). For example, several authors showed that the shape of the occupancy distribution depends on the size of the sample unit and the number of units sampled (Gleason 1929, Curtis and McIntosh 1950, Williams 1950, 1964, McIntosh 1962, Nee et al. 1991). Furthermore, Preston (1948) and Williams $(1950,1964)$ claimed that the bimodality of a frequency distribution is basically a direct consequence of a log-normal (Preston) or of a logarithmic (Williams) abundance distribution. Specifically, Preston's approach gained attention due to its theoretical backing (Golicher et al. 2006) and the fact that log-normal abundance distributions seem to fit a large variety of ecological data sets (Whittaker 1965, May 1975, Lobo and Favila 1999, Gaston and Blackburn 2000, Magurran and Henderson 2003, see also Dewdney 1998, 2000 and Golicher et al. 2006). Papp and Izsak (1997) confirmed the findings of Preston and Williams when they obtained bimodal occupancy distributions as a result of numerical simulations based on log-normal and logarithmic abundance distribu- tions. However, the results of numerical simulations are of limited significance since they usually do not consider the intraspecific aggregation of individuals that may also influence the shape of the occupancy distributions (Williams 1950, Goodall 1952, Wright 1991, Hanski et al. 1993, Hartley 1998).

Despite the general awareness that the method of sampling may influence the shape of an occupancy distribution, the extent to which sampling artefacts can impact apparent patterns has obviously been underestimated. For example, in many studies that focus on the relationship between the spatial extent of the study and the shape of the occupancy distribution, the size and/or number of sample units and spatial extent were simultaneously changed (Collins and Glenn 1990, 1997, Guo et al. 2000, Perelman et al. 2001, Storch and Sizling 2002, Bossuyt et al. 2004, Gibson et al. 2005, Keeley et al. 2005, Heino and Virtanen 2006). In contrast, Heatherly et al. (2007) explicitly kept the sampling intensity constant while varying the spatial extent, whereas Van Rensburg et al. (2000) equalised the sample numbers of all spatial scales by randomly reducing the larger sample numbers to the level of the smallest one. Hence, in the former studies, the relationship between spatial extent and the shape of the occupancy distribution may be masked by the impact of the different sizes and/or numbers of sample units used. This underestimation may be due to the fact that the relative importance of artefactual versus biological mechanisms in terms of shaping occupancy distributions is still largely undetermined (McGeoch and Gaston 2002). Furthermore, studies investigating the effects of artefactual factors on the shape of occupancy distributions are primarily comprised of theoretical considerations and/or numerical simulation studies that assume a random distribution of individuals (Gleason 1929, Preston 1948, Williams 1950, 1964, McIntosh 1962, Nee et al. 1991, Papp and Izsak 1997, Hui and McGeoch 2007). Evidence from empirical data separating the various artefactual influences on the shape of occupancy distributions is scarce. Moreover, the underlying mechanisms that generate changes in the shape of occupancy distributions as a result of varying sampling protocol have not been detailed to date.

In this study, we evaluated the importance of the different and interacting artefactual effects on the shape of occupancy distributions. Using Tokeshi's (1992) test procedure, we first determined the shape of the occupancy distributions for 35 different plant communities that are dominated by perennial, herbaceous species. Then, in the form of a case study, we determined the separate effects from the sampling protocol parameters on the shape of the occupancy distribution in a temperate meadow. The investigated sampling protocol parameters included the size and number of sample units, sample extent, coverage, and intensity. In this study, the underlying mechanisms were identified by tracking the shifts in individual species between occupancy classes with changing sampling 
protocol parameters. Finally, we examined the relationship between log-normal species abundance distributions and the shape of occupancy distributions as well as the influence of different levels of intraspecific aggregation on occupancy distributions by means of artificial species assemblages.

\section{Methods}

\section{Study sites and vegetation data}

This study refers to the spatial scale of individual plant communities, i.e. concrete, local stands of vegetation that are generally distinct from the surrounding vegetation and visually uniform in structure. We determined the shape of the occupancy distribution for 35 communities, including predominantly natural plant communities in the alpine zone in the Bernese Oberland (central Swiss Alps) and semi-natural meadow and pasture communities from different regions in Switzerland. To this end, ten $1 \mathrm{~m}^{2}$ sample quadrats were placed within the uniform area of each community, and all vascular plant species present in a quadrat were recorded.

The case study was performed within a semi-natural meadow on a south/southeastern facing $27^{\circ}$ slope at an elevation of $560 \mathrm{~m}$ a.s.l. in Bremgarten near Bern (Swiss Midlands; $\left.46.58^{\circ} \mathrm{N}, 7.26^{\circ} \mathrm{E}\right)$. The study site is mown by local farmers once per year at the beginning of July. It has not been fertilized since 1958 and is of rather low productivity. This sward is relatively open and dominated by Bromus erectus (nomenclature source: Aeschimann and Heitz 1996). Other frequent and abundant species are Arrhenatherum elatius, Festuca rubra, Achillea millefolium, Centaurea jacea, Galium album, Hippocrepis comosa, Rumex acetosa, and Salvia pratensis. Within a visually uniform area of about $4000 \mathrm{~m}^{2}(80 \times 50 \mathrm{~m})$, we established ten regularly spaced sets of nested quadrats ranging from 0.01 to 100 $\mathrm{m}^{2}$ (Fig. 1). Forty additional $0.25 \mathrm{~m}^{2}$ quadrats were placed in between the previously mentioned quadrats, resulting in a total of 50 quadrats with a size of $0.25 \mathrm{~m}^{2}$ each. We recorded the number of vascular plant species that were present in each quadrat. This data was used to determine

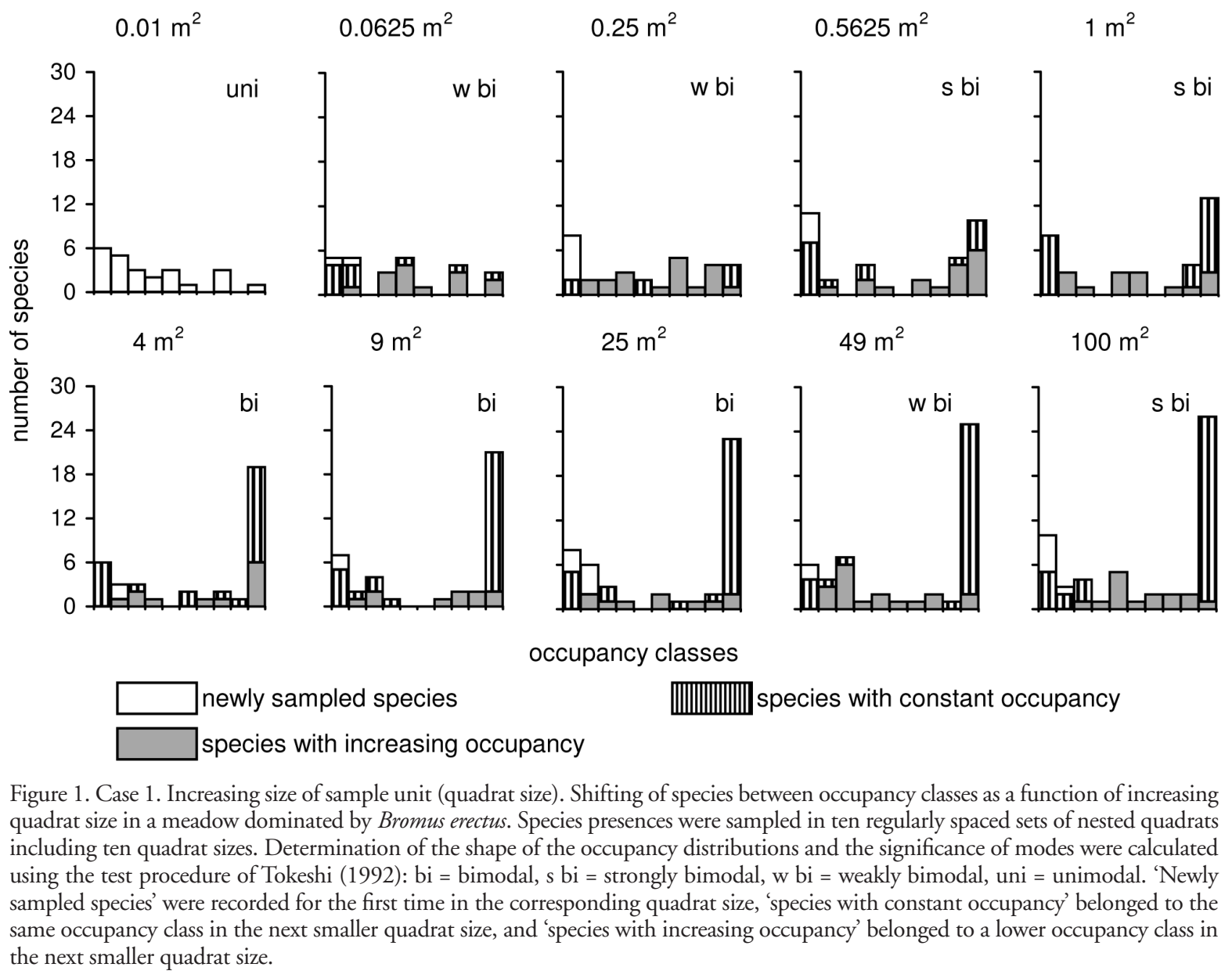


the effect of sample unit size (i.e. nested quadrats), sample number, extent, cover, and intensity, all of which are based on the $500.25 \mathrm{~m}^{2}$ quadrats, on the shape of occupancy distributions (Table 1). Following McGeoch and Gaston (2002, p. 319), sampling coverage (SC) was defined as SC $=(\mathrm{g} \times \mathrm{n}) \mathrm{e}^{-1}$, where $\mathrm{g}$ represents the size of the sample unit (i.e. grain), $\mathrm{n}$ represents the number of sample units, and e represents the sample extent (i.e. the study area). When using the formula for sampling intensity (SI) suggested by McGeoch and Gaston (2002) (i.e. SI $=$ SC $\times$ n), SI increases in cases where sample extent and sample number rise simultaneously. In our view, however, SI should remain constant in these cases. We, therefore, changed the formula for SI as follows: $\mathrm{SI}=\mathrm{SC} \times\left(\mathrm{n} \times \mathrm{e}^{-1}\right)$. In order to determine the influence of sample extent and coverage on the shape of the occupancy distribution, the community area was divided into five sub-areas of $800 \mathrm{~m}^{2}$ (i.e. $16 \times$ $50 \mathrm{~m}$ ) containing ten $0.25 \mathrm{~m}^{2}$ quadrats each. Then, beginning at the western end of the community, the area was progressively enlarged, resulting in a nested design of sub-areas.

\section{Assessment of the shape of the occupancy distributions}

In a majority of the studies, the shapes of the occupancy distribution diagrams were assessed visually. Tokeshi (1992) presented an exact probability method for testing the significance of modes as well as the general shape of an occupancy distribution diagram. Following the recommendation of McGeoch and Gaston (2002), we used this method to assess the shape of the occupancy distributions. Based on a multinomial distribution, the test procedure first calculates the probability $\left(\mathrm{p}_{\mathrm{c}}\right)$ that both the lowest and the highest occupancy classes contain the number of species observed (or even more), thereby assuming a random distribution of species. If $\mathrm{p}_{c}<0.05$, the occupancy distribution is considered either uni- or bimodal; otherwise, it has another shape. Then, in the second step, the probabilities that the lowest and the highest occupancy classes, i.e. $\mathrm{p}_{l}$ and $\mathrm{p}_{\mathrm{h}}$, respectively, contain the number of species observed (or more) are calculated separately. If $\mathrm{p}_{1}<$ 0.5 and $\mathrm{p}_{\mathrm{h}}<0.5$, the occupancy distribution is considered bimodal; otherwise, it is unimodal with the mode in the lowest class if $\mathrm{p}_{\mathrm{h}} \geq 0.5$ or in the highest occupancy class if $\mathrm{p}_{1} \geq 0.5$.

\section{Assessment of the species abundance distributions}

Cover data was available for the B. erectus-meadow, for the five T. flavescens-meadows, and for five sub-alpine meadows and pastures. In these stands, species abundances were sampled in ten $1 \mathrm{~m}^{2}$ quadrats using the point quadrat method. A metal frame similar to the one proposed in Mueller-Dombois and Ellenberg (1974) was used. The needle, measuring $2 \mathrm{~mm}$ in diameter, was lowered vertically through the vegetation canopy at 100 evenly spaced points per $1 \mathrm{~m}^{2}$ quadrat. We counted every resulting needle-plant contact as a measure for 'spatial cover' of each species. The absolute cover values were divided by the total number of hits per $\mathrm{m}^{2}$ to account for relative cover values. As an exception, the species abundances in the B. erectus-meadow at Bremgarten were sampled by visual cover estimation. For this purpose, the $1 \mathrm{~m}^{2}$ quadrats were sub-divided into 16 sub-plots in order to increase the accuracy of the species cover estimation. Relative cover of each species was visually estimated separately for each sub-plot. Then, the average relative cover value of the 16 sub-plots was calculated.

In order to determine whether the abundances of the species of a community were distributed log-normally, we fitted a log-normal distribution to the observed abundance data. To this end, species abundances were classified in logarithmic classes $\left(2^{\mathrm{x}}, \mathrm{x}=0,1,2 \ldots\right)$, which are also called 'octaves' (Williams 1950, 1964). Peripheral classes were pooled in order for each class to hold at least five species. Since unrepresented species are unobservable, i.e. the abundance value 'zero' does not exist, we fitted a truncated log-normal distribution to our data following the proce-

Table 1. The different cases of sampling protocol parameters influencing the shape of the occupancy distributions, that were separately investigated in this study. Bold font: target parameter changed; normal font: parameter hold constant or changing automatically with target parameter changed.

\begin{tabular}{lccccc}
\hline Case & $\begin{array}{c}\text { Size of } \\
\text { sample unit } \\
\mathrm{g}\end{array}$ & $\begin{array}{c}\text { Number of } \\
\text { sample units }\end{array}$ & $\begin{array}{c}\text { Sample extent } \\
(\text { study area })\end{array}$ & $\begin{array}{c}\text { Sample } \\
\text { coverage } \\
\mathrm{SC}=(\mathrm{g} \times \mathrm{n}) \mathrm{e}^{-1}\end{array}$ & $\begin{array}{c}\text { Sample } \\
\text { intensity } \\
\mathrm{SI}=\mathrm{SC} \times\left(\mathrm{n} \times \mathrm{e}^{-1}\right)\end{array}$ \\
\hline 1 & increase & constant & constant & increase & increase \\
2 & constant & increase & constant & increase & increase \\
3 & constant & increase & increase & constant & constant \\
4 & constant & constant & increase & decrease & decrease \\
5 & decrease & increase & constant & constant & increase \\
\hline
\end{tabular}


dure described in Pielou (1975). The goodness of fit of the expected to the observed values was determined using a $\chi^{2}$ test.

\section{Artificial assemblages}

In order to ascertain the influence of the type of species abundance distribution and the degree of individual aggregation on the shape of the occupancy distributions, we performed numerical simulations with artificial assemblages. Each artificial assemblage consisted of $10000(100 \times 100)$ cells corresponding to the same number of individuals. The abundances (i.e. the number of individuals) of all species of an artificial assemblage were deliberately selected in order to produce different abundance distribution patterns (Fig. 5, top line) based on logarithmic abundance classes $\left(2^{\mathrm{x}}, \mathrm{x}=0,1,2,3, \ldots ;\right.$ ' 'octaves' sensu Williams 1950,1964$)$. The following abundance distribution patterns were created: an assemblage with a uniform abundance distribution (A), a bimodal distribution with the modes in the extreme abundance classes (B), and three unimodal distributions with the mode in the medium class $(\mathrm{C})$, in the lowest class (D), and in the highest abundance class (E). On the basis of an arithmetic scale, all artificial abundance distributions (i.e. A to E) have the shape of a reversed 'J', which is also known as a 'hollow-curve'; i.e. the number of species with low abundance is much higher than the number of species with higher abundances.

To generate the occupancy distributions, the positions of ten regularly spaced sets of nested sampling plots were defined within the frame of $100 \times 100$ cells. The sets consisted of nested plots of $3 \times 3,5 \times 5,7 \times 7$, and $10 \times 10$ cells with one corner in common. The artificial individuals (i.e. those belonging to different artificial species) of the five artificial assemblages (i.e. A to E) were distributed randomly within the frame, and the presence of each species in the plots was counted. This procedure was repeated 20 times for each artificial assemblage, and the mean number of species per occupancy class was computed.

Artificial assemblages with different levels of intraspecific aggregation of individuals were generated using the subsequent procedure. First, 200, 300, or 400 aggregates (i.e. $3 \times 3$ cells each) were distributed randomly within the frame while avoiding overlapping. Then, a certain proportion of the individuals of the 14 most abundant species in artificial assemblage $\mathrm{A}$ (i.e. the uniform abundance distribution, Fig. 5) was distributed randomly on the aggregates; i.e. ca $23 \%$ of the individuals of the most abundant species were distributed on 200 aggregates, $34 \%$ on 300 aggregates, and $46 \%$ on 400 aggregates. Hence, each aggregate consisting of $3 \times 3$ cells was exclusively occupied by one species. Next, the remaining individuals of the most abundant species and the individuals of the less abundant species were distributed randomly on the remaining vacant cells. Finally, the number of each species present in the ten plots was counted. This procedure was repeated 20 times for each degree of aggregation, and the mean number of species per occupancy class was computed.

\section{Results}

For convenience, we adopted Hanski's (1982a) core and satellite terminology in the following text without implying the corresponding biological mechanisms: core species refers to an occupancy higher than 0.9 , and satellite species refers to an occupancy less than or equal to 0.1 .

\section{Shape of the occupancy distributions}

With a quadrat size of $1 \mathrm{~m}^{2}, 30$ out of 35 plant communities exhibited a significant mode in the lowest occupancy class; among them, 20 communities had a second mode in the highest class; i.e. they demonstrated a bimodal occupancy distribution (Table 2). For five plant communities, neither a satellite nor a core mode was detected. In 21 communities, the highest mode occurred in the satellite class, and in the remaining eight, the highest mode occurred in the core class.

\section{Case 1. Increasing size of sample unit (quadrat size)}

Increasing the quadrat size resulted in the following three effects (Fig. 1): 1) new species were sampled and generally entered the satellite class; 2) many species with relatively low occupancy shifted to classes of higher occupancy; and finally, 3) many species reached the core class and remained there when the quadrat size was further enlarged. Consequently, the total number of species sampled and the number of core species gradually increased with the enlargement of quadrat size following a logarithmic curve, while the number of satellite species fluctuated independent of the quadrat size (Fig. 2).

The shape of the occupancy distribution underwent an obvious transformation with increasing quadrat size (Fig. 1). The smallest quadrat size $\left(0.01 \mathrm{~m}^{2}\right)$ had a unimodal, right-skewed distribution. Between quadrat sizes of 0.0625 and $0.5625 \mathrm{~m}^{2}$, the distribution became increasingly bimodal with the larger mode still in the satellite class. Between quadrat sizes of 0.5625 and $1 \mathrm{~m}^{2}$, the largest mode switched from the left to the right; i.e. the core class contained more species than the satellite class. In quadrat sizes of $1 \mathrm{~m}^{2}$ and larger, the occupancy distribution remained generally bimodal with a distinctly larger mode in the core class.

Seven species switched from the satellite class to the core class in the range of enlarging quadrat sizes in this study (Table 3). For example, Poa pratensis occurred in one 
Table 2. Determination of the shape of the occupancy distributions and the significance of modes of 35 plant communities using the test procedure by Tokeshi (1992). Ten quadrats per community, quadrat size $1 \mathrm{~m}^{2} \cdot \mathrm{n}=$ total number of species recorded in the ten plots; $\mathrm{n}_{1}=$ number of species in the satellite class, $\mathrm{n}_{\mathrm{h}}=$ number of species in the core class; $\mathrm{p}_{\mathrm{c}}=$ probability of obtaining the observed occupancies of the satellite and core class combined; $\mathrm{p}_{1}=$ probability of obtaining the observed occupancy in the satellite class; $\mathrm{p}_{\mathrm{h}}=$ probability of obtaining the observed occupancy in the core class; mode: largest mode in the satellite $(\mathrm{L})$ or in the core class $(\mathrm{H})$, respectively.

\begin{tabular}{|c|c|c|c|c|c|c|c|c|}
\hline Community & $\mathrm{n}$ & $\mathrm{n}_{1}$ & $\mathrm{n}_{\mathrm{h}}$ & $\mathrm{p}_{\mathrm{c}}$ & $\mathrm{p}_{1}$ & $\mathrm{p}_{\mathrm{h}}$ & Shape & Mode \\
\hline Dryas octopetala - rock comm. & 35 & 4 & 2 & 0.403 & 0.469 & 0.878 & other & \\
\hline Dryas octopetala - scree comm. & 20 & 3 & 2 & 0.180 & 0.323 & 0.608 & other & \\
\hline Thlaspi repens - scree comm. 1 & 22 & 10 & 1 & 0.000 & 0.000 & 0.902 & unimodal & $\mathrm{L}$ \\
\hline Thlaspi repens - scree comm. 2 & 8 & 1 & 2 & 0.092 & 0.570 & 0.187 & other & \\
\hline Leontodon montanus - scree comm. & 12 & 2 & 2 & 0.099 & 0.341 & 0.341 & other & \\
\hline Polytrichum sexangularis - snowbed comm. & 13 & 2 & 1 & 0.269 & 0.379 & 0.746 & other & \\
\hline Salix herbacea-snowbed comm. 1 & 24 & 6 & 5 & 0.001 & 0.028 & 0.085 & bimodal & $\mathrm{L}$ \\
\hline Salix herbacea-snowbed comm. 2 & 14 & 4 & 3 & 0.004 & 0.044 & 0.158 & bimodal & $\mathrm{L}$ \\
\hline Salix herbacea-snowbed comm. 3 & 21 & 5 & 6 & 0.000 & 0.052 & 0.014 & bimodal & $\mathrm{H}$ \\
\hline Salix herbacea-snowbed comm. 4 & 23 & 6 & 7 & 0.000 & 0.023 & 0.006 & strongly bimodal & $\mathrm{H}$ \\
\hline Arabis caerulea - snowbed comm. & 37 & 8 & 4 & 0.011 & 0.027 & 0.514 & unimodal & $\mathrm{L}$ \\
\hline Saxifraga androsacea - snowbed comm. & 33 & 8 & 4 & 0.004 & 0.014 & 0.423 & weakly bimodal & $\mathrm{L}$ \\
\hline Carex curvula - grassland & 27 & 7 & 2 & 0.010 & 0.015 & 0.767 & unimodal & $\mathrm{L}$ \\
\hline Carex firma - grassland 1 & 35 & 14 & 2 & 0.000 & 0.000 & 0.878 & unimodal & $\mathrm{L}$ \\
\hline Carex firma - grassland 2 & 23 & 4 & 8 & 0.000 & 0.193 & 0.001 & bimodal & $\mathrm{H}$ \\
\hline Carex/Sesleria - grassland 1 & 60 & 16 & 2 & 0.000 & 0.000 & 0.986 & strongly unimodal & $\mathrm{L}$ \\
\hline Carex/Sesleria - grassland 2 & 45 & 10 & 11 & 0.000 & 0.012 & 0.004 & strongly bimodal & $\mathrm{H}$ \\
\hline Carex/Sesleria - grassland 3 & 40 & 14 & 6 & 0.000 & 0.000 & 0.206 & bimodal & $\mathrm{L}$ \\
\hline Carex/Sesleria - grassland 4 & 58 & 9 & 10 & 0.004 & 0.121 & 0.060 & bimodal & $\mathrm{H}$ \\
\hline Carex ferruginea - grassland & 69 & 19 & 0 & 0.000 & 0.000 & 1.000 & strongly unimodal & $\mathrm{L}$ \\
\hline Elyna myosuroides - grassland 1 & 34 & 11 & 4 & 0.000 & 0.000 & 0.446 & weakly bimodal & $\mathrm{L}$ \\
\hline Elyna myosuroides - grassland 2 & 46 & 11 & 8 & 0.000 & 0.005 & 0.084 & bimodal & $\mathrm{L}$ \\
\hline Nardus stricta - grassland & 61 & 21 & 13 & 0.000 & 0.000 & 0.007 & strongly bimodal & $\mathrm{L}$ \\
\hline Bromus erectus - pasture & 69 & 15 & 9 & 0.000 & 0.003 & 0.250 & weakly bimodal & $\mathrm{L}$ \\
\hline Bromus erectus - meadow & 36 & 8 & 13 & 0.000 & 0.024 & 0.000 & strongly bimodal & $\mathrm{H}$ \\
\hline Molinia caerulea - meadow & 37 & 5 & 5 & 0.081 & 0.309 & 0.309 & weakly bimodal & \\
\hline Trisetum flavescens - meadow 1 & 32 & 9 & 6 & 0.000 & 0.003 & 0.094 & bimodal & $\mathrm{L}$ \\
\hline Trisetum flavescens - meadow 2 & 56 & 6 & 12 & 0.003 & 0.493 & 0.008 & weakly bimodal & $\mathrm{H}$ \\
\hline Trisetum flavescens - meadow 3 & 34 & 5 & 10 & 0.000 & 0.250 & 0.001 & bimodal & $\mathrm{H}$ \\
\hline Trisetum flavescens - meadow 4 & 70 & 18 & 4 & 0.000 & 0.000 & 0.929 & unimodal & $\mathrm{L}$ \\
\hline Trisetum flavescens - meadow 5 & 48 & 9 & 8 & 0.003 & 0.046 & 0.102 & bimodal & $\mathrm{L}$ \\
\hline Festuca violacea - grassland & 55 & 12 & 2 & 0.007 & 0.007 & 0.978 & strongly unimodal & $\mathrm{L}$ \\
\hline Adenostyles alliariae - tall herb comm. & 58 & 22 & 1 & 0.000 & 0.000 & 0.998 & strongly unimodal & $\mathrm{L}$ \\
\hline Loiseleuria procumbens - heath 1 & 13 & 4 & 2 & 0.009 & 0.034 & 0.379 & weakly bimodal & $\mathrm{L}$ \\
\hline Loiseleuria procumbens - heath 2 & 45 & 8 & 4 & 0.044 & 0.076 & 0.671 & weakly unimodal & $\mathrm{L}$ \\
\hline
\end{tabular}




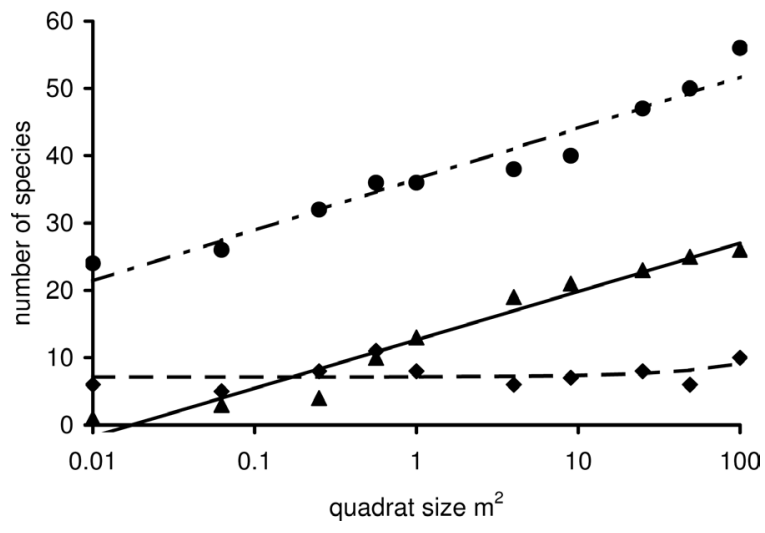

Figure 2. Case 1. Increasing size of sample unit (quadrat size). Total number of species $\left(--, y=3.29 \ln (\mathrm{x})+36.53, \mathrm{R}^{2}=\right.$ $0.942)$, number of core species $(\boldsymbol{\Lambda}-\mathrm{y}=3.12 \ln (\mathrm{x})+12.64$, $\left.\mathrm{R}^{2}=0.957\right)$, and number of satellite species $(---, \mathrm{y}=0.02 \mathrm{x}$ $\left.+7.14, \mathrm{R}^{2}=0.105\right)$ as a function of quadrat size in a meadow dominated by Bromus erectus. Species presences were sampled in ten regularly spaced sets of nested quadrats including ten quadrat sizes.

$0.25 \mathrm{~m}^{2}$ quadrat; however, in quadrat sizes of $4 \mathrm{~m}^{2}$ and larger, this species was found in all ten quadrats.

\section{Case 2. Increasing number of sample units (sample number)}

Increasing the number of sampled quadrats within the same area resulted in the following (Fig. 3a): 1) new species were sampled and mostly were classified in the satellite class; 2) species with relatively low occupancy shifted to classes of higher occupancy if their occupancy was higher in the additionally sampled quadrats as compared to the original quadrats; and 3) in contrast, species with relatively high occupancy shifted to classes of lower occupancy if their occupancy was lower in the additionally sampled quadrats. As a result, the number of core species remained generally constant, whereas the total number of sampled species and the number of satellite species gradually increased with rising sample number. The shape of the occupancy distribution changed slightly with increasing quadrat number from a weakly bimodal distribution for 10 and 20 quadrats to a unimodal distribution with a satellite mode for 30, 40, and 50 quadrats (Fig. 3a).

\section{Cases 3 and 4. Increasing sample extent (study area)}

Increasing the area sampled and the number of quadrats (case 3, Fig. 3b) lead to very similar results as those obtained when solely increasing the number of sample units (i.e. case 2).

Keeping the number of quadrats constant while increasing the sample area (case 4) resulted in the following effects (Fig. 3c): 1) the sampling of new quadrats within the larger area lead to the inclusion of new species; 2 ) the numbers of species that increased and those that decreased were approximately equivalent to one another; and 3) the number of satellite and core species were generally stable. Overall, the shape of the occupancy distribution remained unchanged, i.e. bimodal with the highest mode in the satellite class.

\section{Case 5. Increasing sample intensity}

Sampling the same surface area with more but smaller quadrats means an increase in sample intensity. In this case, the number of satellite species increases, while the number of core species decreases. For example, sampling a $10 \mathrm{~m}^{2}$ area with forty $0.25 \mathrm{~m}^{2}$ quadrats resulted in 14 satellite and four core species, while recording the same area with ten $1 \mathrm{~m}^{2}$ quadrats yielded only eight satellite species but 13 core species (Fig. 4). Thus, increasing the

Table 3. Species switching from the satellite to the core class with increasing quadrat size. The numbers indicate the number of quadrats occupied by the species (ten quadrats per size were sampled). Bold: satellite (1) and core (10) species.

\begin{tabular}{lcccccccccc}
\hline & \multicolumn{1}{c}{ Quadrat size $\left(\mathrm{m}^{2}\right)$} & \multicolumn{1}{c}{} & \\
Species & 0.01 & 0.0625 & 0.25 & 0.5625 & 1 & 4 & 9 & 25 & 49 & 100 \\
\hline Dactylis glomerata & 0 & 0 & 0 & 1 & 1 & 6 & 9 & 9 & 9 & 10 \\
Lotus corniculatus & 1 & 2 & 4 & 4 & 5 & 10 & 10 & 10 & 10 & 10 \\
Poa pratensis & 0 & 0 & 1 & 2 & 5 & 10 & 10 & 10 & 10 & 10 \\
Ranunculus bulbosus & 1 & 1 & 2 & 4 & 6 & 7 & 8 & 9 & 10 & 10 \\
Salvia pratensis & 1 & 4 & 7 & 10 & 10 & 10 & 10 & 10 & 10 & 10 \\
Sanguisorba minor & 0 & 1 & 4 & 4 & 5 & 8 & 10 & 10 & 10 & 10 \\
Silene nutans & 0 & 0 & 1 & 1 & 2 & 4 & 4 & 8 & 10 & 10 \\
\hline
\end{tabular}


a) Case 2: Increasing number of sample units (quadrats), constant sample extent

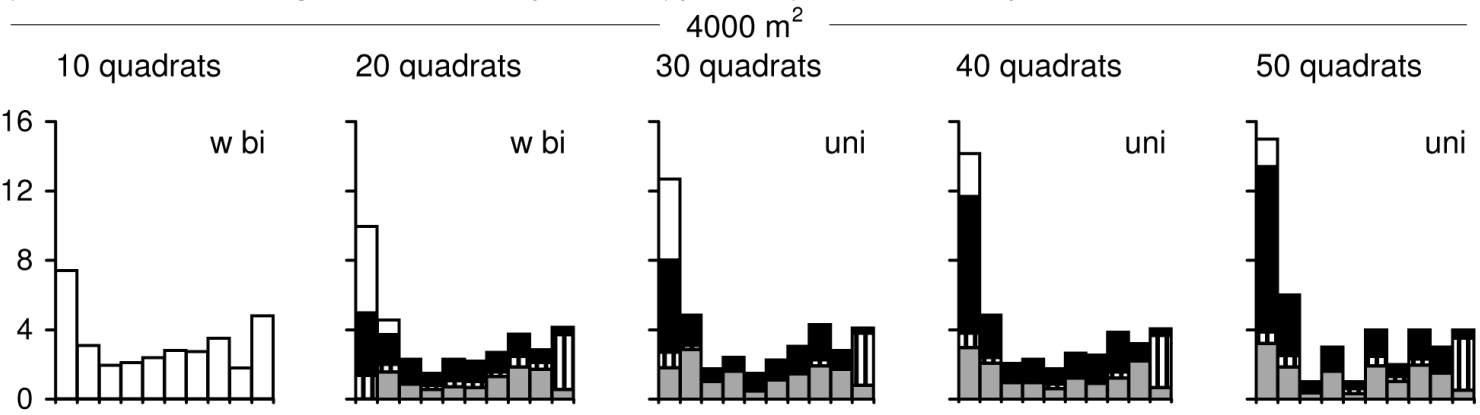

b) Case 3: Increasing sample extent (study area), increasing number of sample units
$800 \mathrm{~m}^{2}$
$1600 \mathrm{~m}^{2}$
$2400 \mathrm{~m}^{2}$
$3200 \mathrm{~m}^{2}$
$4000 \mathrm{~m}^{2}$
10 quadrats
20 quadrats
30 quadrats
40 quadrats
50 quadrats
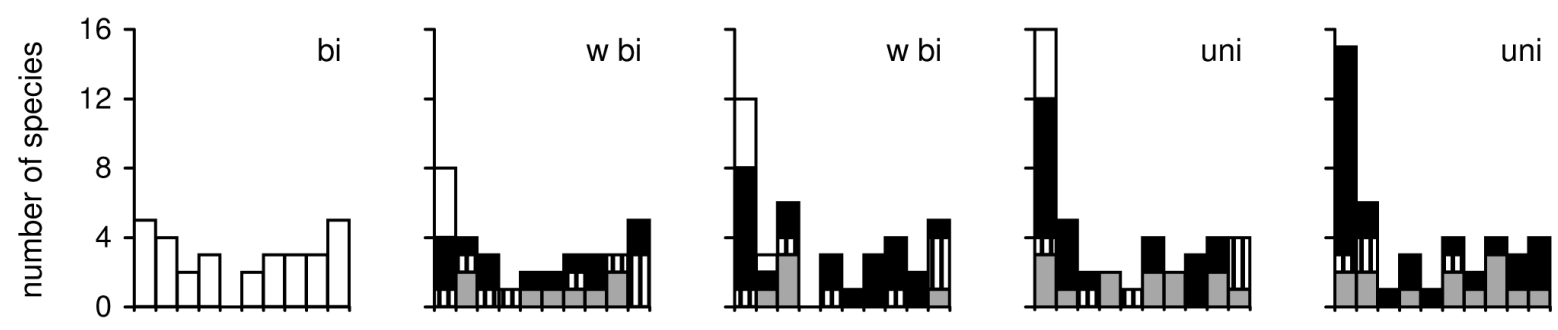

c) Case 4: Increasing sample extent, constant number of sample units

$800 \mathrm{~m}^{2} \quad 1600 \mathrm{~m}^{2}$
$2400 \mathrm{~m}^{2}$ 10 quadrats

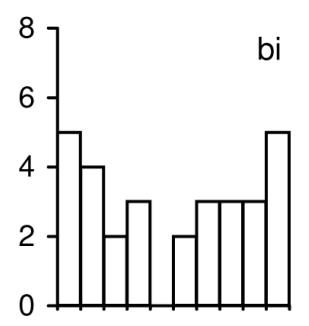

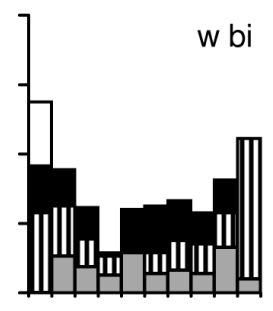

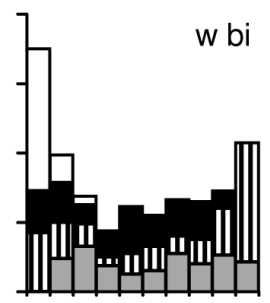

occupancy classes
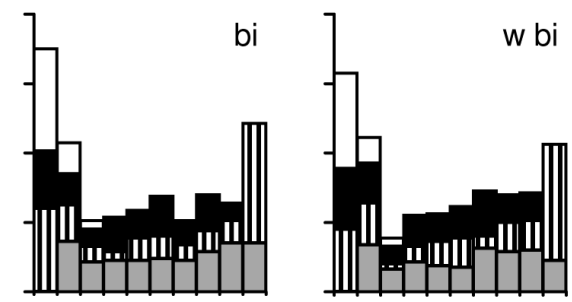

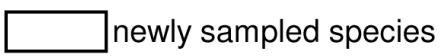

species with increasing occupancy

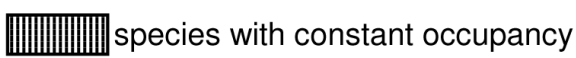

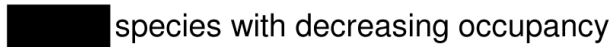

Figure 3. Shifting of species between occupancy classes as a function of increasing number of sample units (a and b) and increasing sample extent (b and c) in a meadow dominated by Bromus erectus. Species presences were sampled in regularly spaced $0.25 \mathrm{~m}^{2} \mathrm{quad}-$ rats. For a), 10, 20, 30, or 40 quadrats were randomly selected out of the 50 quadrats available and species occupancy of each selection was computed. This procedure was repeated 20 times and mean number of species per occupancy class are depicted in the occupancy distribution diagrams. For b), all quadrats lying within each sample extent were recorded. For c), ten quadrats were randomly selected out of the 20,30, 40, or 50 quadrats available (depending on sample extent) and species occupancy of each selection was computed. This procedure was repeated 20 times and mean number of species per occupancy class are depicted. Determination of the shape of the occupancy distributions and the significance of modes were calculated using the test procedure of Tokeshi (1992). For legend see Fig. 1, in addition, 'species with decreasing occupancy' belonged to a higher occupancy class in the next smaller sample number or sample extent, respectively. 


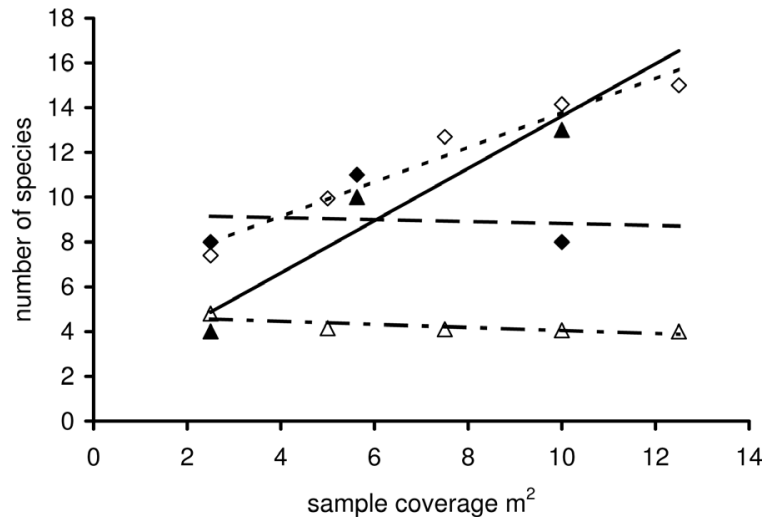

Figure 4. Case 5. Sample intensity. Response of the number of satellite species and the number of core species to changes in size and number of sample units (quadrats) within the same sample coverage. Legend: response of the number of satellite species to change in quadrat size $\left(---, y=-0.04 x+9.27, \mathrm{R}^{2}=0.009\right)$ and number of quadrats $\left(\nabla \ldots, y=0.77 x+6.02, R^{2}=0.956\right)$; response of the number of core species to change in quadrat size $\left(\mathbf{\Lambda}-, \mathrm{y}=1.16 \mathrm{x}+1.94, \mathrm{R}^{2}=0.920\right)$ and number of quadrats $\left(\triangle---, y=-0.06 x+4.73, R^{2}=0.667\right)$.

sampling intensity by recording the same area with more but smaller quadrats changes the shape of the occupancy distribution from a strongly bimodal distribution with the higher mode in the core class (Fig. $1,1 \mathrm{~m}^{2}$ ) to a unimodal distribution with the higher mode in the satellite class (Fig. 3a, 40 quadrats).

\section{Species abundance distributions}

Ten out of 11 species abundance distributions did not significantly (i.e. $\mathrm{p} \leq 0.05$ ) differ from a log-normal distribution (Table 4). However, only five of these communities generated a bimodal occupancy distribution. Species abundances in Trisetum-meadow 5 were not log-normally distributed; nevertheless, this stand exhibited a bimodal occupancy distribution.

The abundance distributions of the artificial assemblages $\mathrm{A}, \mathrm{C}$, and $\mathrm{E}$ were not significantly different from lognormal distributions (Table 4). A and C produced occupancy distribution patterns that correspond to those observed in reality (Fig. 5), i.e. unimodal distributions with a satellite mode in small plot sizes becoming bimodal with increasing plot size. The occupancy distribution of the artificial assemblage E, however, was not equivalent to real plant communities. The species abundances of the artificial assemblages $\mathrm{B}$ and $\mathrm{D}$ were obviously not log-normally distributed. Nevertheless, their occupancy distributions were similar to those observed in reality and also included bimodality.

\section{Intraspecific aggregation of individuals}

With the scale of 49 cells per plot, a bimodal occupancy distribution switched to a unimodal, right-skewed distribution when individuals were aggregated (Fig. 6). In the other plot sizes, the general pattern of the occupancy distributions did not fundamentally change with the degree of aggregation of individuals. However, the slope of the occupancy distributions of the two smallest plot sizes (i.e. 9 and 25 cells) became slightly steeper with an increasing degree of aggregation. Generally, the number of core species decreased with an increasing level of aggregation.

\section{Discussion}

\section{Shape of the occupancy distributions}

Our results correspond with the body of studies showing that occupancy distributions with one single mode, i.e. the satellite mode, or with two modes in the extreme occupancy classes are common patterns in ecological communities (Tokeshi 1992, Scheiner and Rey-Benayas 1997, McGeoch and Gaston 2002). Other types of occupancy distributions without these modes occurred in our data, but they seem to be restricted to early successional plant communities. In three communities, the low number of species may have impeded the identification of the satellite and/or core mode when using Tokeshi's (1992) test procedure. However, these statements have to be qualified by considering that the shapes of the occupancy distributions listed in Table 2 are the direct result of the chosen sampling protocol, especially the size of the sampling units $\left(1 \mathrm{~m}^{2}\right)$.

\section{Cases 1 and 2. Increasing size and number of sample units (quadrats)}

Indeed, the shape of the occupancy distribution diagram characteristically changes with the higher mode switching from the satellite to the core class with increasing quadrat size (Fig. 1). The appearance of the core mode and its increase with increasing quadrat size are likely a direct consequence of the exponential relationship between density and occupancy as suggested by Ashby (1935). In accordance with Ashby's assertions, the same density of a species produces higher occupancy values in large quadrats as compared to that in small ones. This means that the species shifts to a higher occupancy class with increasing quadrat size as a result of additionally sampled individuals of the species. Then, the species accumulate in the highest occupancy class, which also has the broadest density range (Greig-Smith 1964). Consequently, the number of species in this class will constantly rise with increasing quadrat size 
Table 4. Goodness of fit $\left(\chi^{2}\right.$ test, $\left.\mathrm{p}<0.05\right)$ of the expected values of a truncated log-normal distribution to the distribution of observed cover values of the species in 11 plant communities (ten quadrats per community, quadrat size $1 \mathrm{~m}^{2}$ ), and to the distribution of individual numbers of the species of five artificial assemblages, respectively. The fitting was performed following the procedure given in Pielou (1975). The fifth column indicates the shape of the occupancy distribution for the plant communities using the test procedure of Tokeshi (1992).

\begin{tabular}{|c|c|c|c|c|c|}
\hline \multirow[b]{2}{*}{ Community } & \multicolumn{4}{|c|}{ Pielou-test } & \multirow{2}{*}{$\begin{array}{l}\text { Tokeshi-test } \\
\text { Occupancy } \\
\text { distribution }\end{array}$} \\
\hline & DF & $\chi^{2}$ & $\mathrm{p}$ & $\begin{array}{l}\text { Abundance } \\
\text { distribution }\end{array}$ & \\
\hline Bromus erectus-meadow & 2 & 2.877 & 0.237 & log-normal & strongly bimodal \\
\hline Trisetum flavescens-meadow 1 & 1 & 0.101 & 0.751 & log-normal & bimodal \\
\hline Trisetum flavescens-meadow 2 & 4 & 1.051 & 0.902 & log-normal & weakly bimodal \\
\hline Trisetum flavescens-meadow 3 & 1 & 0.705 & 0.401 & log-normal & bimodal \\
\hline Trisetum flavescens-meadow 4 & 6 & 6.060 & 0.417 & log-normal & unimodal \\
\hline Trisetum flavescens-meadow 5 & 3 & 7.930 & 0.047 & - & bimodal \\
\hline Sub-alpine meadow 1 & 4 & 2.877 & 0.579 & log-normal & bimodal \\
\hline Sub-alpine meadow 2 & 5 & 3.414 & 0.636 & log-normal & unimodal \\
\hline Sub-alpine meadow 3 & 6 & 5.021 & 0.541 & log-normal & unimodal \\
\hline Sub-alpine pasture 1 & 6 & 5.664 & 0.462 & log-normal & strongly unimodal \\
\hline Sub-alpine pasture 2 & 6 & 8.925 & 0.178 & log-normal & unimodal \\
\hline Artificial assemblage A & 8 & 4.570 & 0.802 & log-normal & \multirow{5}{*}{$\begin{array}{l}\text { depends on } \\
\text { plot size } \\
\text { (Fig. 5) }\end{array}$} \\
\hline Artificial assemblage B & 8 & 24.392 & 0.002 & - & \\
\hline Artificial assemblage $\mathrm{C}$ & 8 & 1.075 & 0.998 & log-normal & \\
\hline Artificial assemblage D & 8 & 18.712 & 0.016 & - & \\
\hline Artificial assemblage E & 3 & 1.702 & 0.636 & log-normal & \\
\hline
\end{tabular}

as long as the sample extent remains constant. Thus, the core mode occurs when the sample unit size is relatively large as compared to the sample extent, thereby confirming the corresponding suppositions of Gaston and Blackburn (2000), Guo et al. (2000), and Hui and McGeoch (2007).

The existence and the preservation of a satellite mode with increasing quadrat size are very likely due to a large pool of species with low abundance within the community. The stepwise enlargement of the sample quadrats leads to the recording of additional rare species that keep the number of species in the satellite class high even though some satellite species shift to higher occupancy classes. This finding is confirmed by the only case where no satellite mode was developed, i.e. in the artificial assemblage E, which lacked rare species (Fig. 5). Species abundance distributions describing a hollow-curve on an arithmetic scale, thereby indicating few very abundant and many rare species, seem to be universal (Dewdney 1998, 2000, McGill et al. 2007). Hence, occupancy distributions with a satellite mode due to the large number of rare species may be considered the 'normal' form of an occupancy distribution. This assumption is supported by the fact that the occupancy distributions of 30 out of 35 plant communities in this study exhibited a peak in the satellite class (Table 2). Moreover, Scheiner and Rey-Benayas (1997) found a unimodal occupancy distribution with a satellite mode in 65 data sets when they analysed the distribution patterns of terrestrial plants in 74 landscapes around the globe.

Both increasing the number of sample units within the same sample extent and the enlargement of the sample unit size are possibilities to increase the sample coverage. However, they do not result in the same impact on the shape of the occupancy distribution. When increasing the number of quadrats sampled, the number of core species remains stable, and the number of satellite species rises. The shape of the occupancy distribution changes slightly from weakly bimodal to unimodal with a satellite mode. Thus, increasing the number of quadrats has the opposite effect on the number of satellite and core species when compared to the enlargement of the quadrat size. Although seemingly 


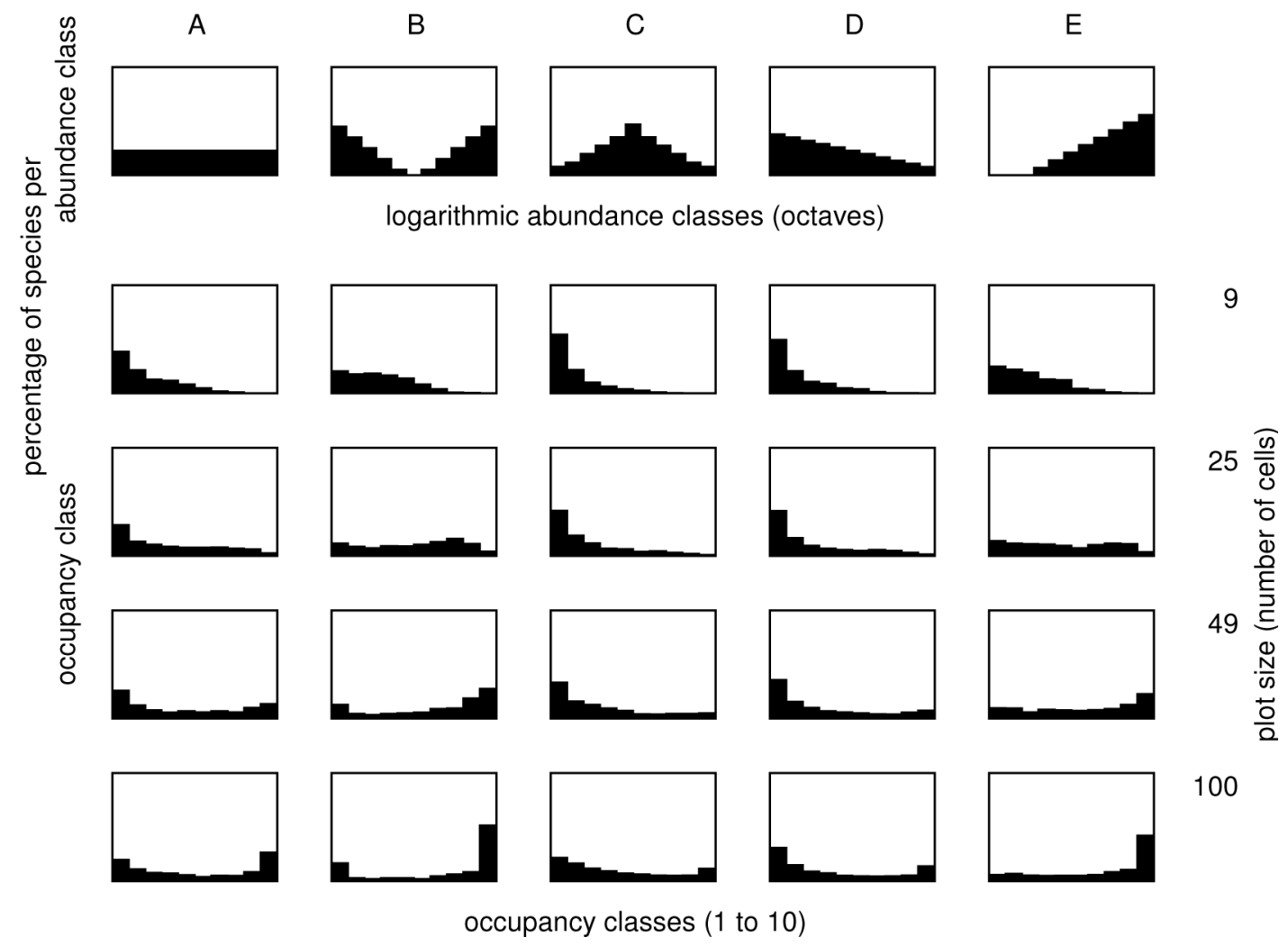

Figure 5. Abundance distributions of the artificial assemblages A to E (top line) and the resulting average occupancy distributions in different plot sizes (number of cells per plot). Mean percentage of species per abundance and occupancy classes are indicated since the number of species is highly different among the five artificial assemblages (for details see text).

confusing, this result can be explained. Since the sample quadrats are spaced apart from one another, an increase in sample number means that new patches within the community habitat are recorded. These new patches may show different environmental conditions and potentially contain species that were not recorded with the smaller number of sample units. The newly sampled species contribute to the increase in the number of satellite species. The number of core species remains generally constant since the number of species newly entering the core class is compensated by the number of species that shift to a lower occupancy class. On the other hand, an increase in quadrat size means that the area adjacent to the original quadrat is sampled. The probability that this area is clearly distinct from the original quadrat in terms of environmental conditions is relatively low (Gaston and Blackburn 2000); therefore, the probability of recording new species is also rather low. The few new species sampled will enter the satellite class, but because species 'automatically' shift to higher occupancy classes with increasing quadrat size, the satellite class also loses some species, which ultimately leads to an almost stable number of satellite species. The number of core species increases as a result of species ac- cumulation in this class. This assertion is confirmed by the fact that the increase in the total sampling area from 2.5 (i.e. ten $0.25 \mathrm{~m}^{2}$ quadrats) to $10 \mathrm{~m}^{2}$ (i.e. forty $0.25 \mathrm{~m}^{2}$ quadrats) resulted in recording about 13 new species (Fig. $3 \mathrm{a})$, whereas the same increase in the total sampling area with increasing quadrat size (from ten $0.25 \mathrm{~m}^{2}$ quadrats to ten $1 \mathrm{~m}^{2}$ quadrats) only resulted in recording four new species (Fig. 1). Thus, an increase in the sample number mostly leads to recording a higher proportion of spatial variability in terms of irregularities in species distribution and heterogeneity in environmental conditions within the community area. Otherwise, an increase in quadrat size does not result in a loss of spatial variability, but a larger proportion of variability is included within a single sample unit and can no longer be differentiated (Wiens 1989).

Overall, these results provide empirical evidence and mechanistic explanations for the findings of several authors who predicted similar effects by changes in the sample unit size and number on the shape of occupancy distributions on the basis of theoretical considerations or numerical simulations (Gleason 1929, Curtis and McIntosh 1950, Goodall 1952, McIntosh 1962, Williams 1950, 1964). 
0

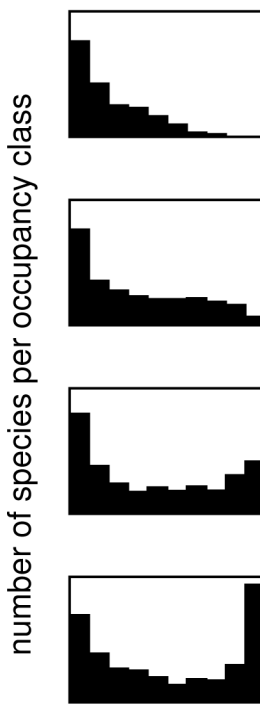

200


occupancy classes (1 to 10 )



400
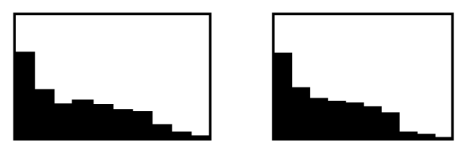

25

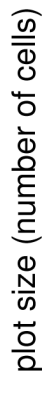

100

Figure 6. Average occupancy distributions of the artificial assemblage A (Fig. 5) as a function of the level of intraspecific individual aggregation and of plot size. Mean numbers of species per occupancy class of 20 repeats are indicated (for details see text).

\section{Cases 3 and 4. Increasing sample extent (study area)}

Increasing the sample extent leads to a higher spatial variability within the sample. In case 3 where the number of sample units increased approximately at the same rate as the sample extent, i.e. the sample coverage is unchanged, the number of satellite species increased, and the number of core species remained constant for the same reasons as explained for case 2 .

Enlarging the sample extent by holding the number of sample units constant (case 4), i.e. the sample coverage decreases, results in several quadrats no longer being sampled. Therefore, species with low occupancy values, especially satellite species, may disappear from the sample. On the other hand, the sampling of new quadrats within the enlarged area leads to the recording of new species. The loss of low-occupancy species and the sampling of new species seem to balance one another, resulting in a generally stable number of species in the satellite class. The constant number of core species with increasing sample extent most likely reflects the situation in which a stable number of high-abundance species with a relatively uniform distribution within the community area is recorded. Since both the satellite and the core mode were maintained with decreasing coverage, the shape of the occupancy distribution did not fundamentally change. Concerning satellite species, our findings are contradictory to the results of Heatherly et al. (2007) in their investigation of the occupancy patterns of stream insects on a regional scale. They found an increase in the proportion of satellite taxa and stronger unimodal occupancy distributions with increasing spatial extent.

\section{Case 5. Increasing sample intensity}

Increasing the sample intensity by sampling the same total area with more but smaller quadrats represents an increase in the sampling resolution; i.e. a higher proportion of spatial variability in terms of irregularities in species distribution and heterogeneity in environmental conditions is recorded. Based on cases 1 and 2, a decrease in quadrat size and a simultaneous increase of quadrat number must lead to a gain in satellite species and to a loss in core species. Consequently, the shape of the occupancy distribution switches from bimodal to unimodal with a satellite mode.

\section{Species abundance distributions}

Based on field data and numerical simulations, our investigation revealed that many types of abundance distributions are able to produce occupancy distributions that 
correspond to those observed in reality. Even abundance distributions that hardly occur in nature may generate left-mode or bimodal occupancy distributions. Thus, Preston's (1948) assertion that the shape of an occupancy distribution is merely the direct consequence of a lognormal abundance distribution of species appears to be unjustified. Furthermore, Fig. 5 shows that the quadrat size seems to be more important in determining the shape of the occupancy distributions than the type of abundance distribution. In fact, four out of five different abundance distributions produced a bimodal occupancy distribution in the largest quadrat size; the fifth generated a unimodal distribution with the mode in the core class. This latter assemblage lacks a high number of low-abundance species that would be needed to form a peak in the satellite class. A species abundance distribution of this form seldom occurs in nature (McGill et al. 2007), nevertheless, a unimodal occupancy distribution with a core mode was found for freshwater bryophytes occupying provinces of Scandinavia (Heino and Virtanen 2006).

\section{Intraspecific aggregation of individuals}

With an increasing degree of aggregation of individuals, the number of species in the higher occupancy classes generally decreases. This may lead to a shift of the shape of the occupancy distribution from bimodal for a random dispersion of individuals to unimodal with a satellite mode for aggregated individuals (plot size 49 in Fig. 6). Green and Plotkin (2007) investigated the effect of intraspecific aggregation of individuals on the shape of different species abundance models, including log-series and log-normal models. They found that intraspecific aggregation skews the abundance distribution towards both more rare and more abundant species in the sample as compared to random dispersion. In the case of the lognormal abundance distribution, the rare skew was more pronounced than the common skew. Consequently, the probability of a species occurring in a sample unit diminishes, resulting in a lower occupancy value for this species. Accordingly, the number of species in the higher occupancy classes is lower, also preventing the occurrence of a core mode. Thus, these results agree with the findings of Hanski et al. (1993), which stated that the number of core species will decrease with aggregated individuals as compared to a random distribution, whereas Goodall's (1952) prediction of an increasing number of satellite species could not be confirmed. In our investigation, the total number of species sampled remained generally constant with an increasing degree of aggregation, which does not agree with Goodall (1952) and Hughes (1986), who predicted a decrease in species number. However, the size of the sample unit seems to have a greater impact on the shape of the occupancy distribution than the degree of aggregation (Fig. 6).

\section{Core and satellite species}

The fact that the shape of the occupancy distributions and the numbers of core and satellite species highly depend on the sample unit size (Fig. 1) creates serious implications for empirical studies. When the sample units represent sub-units of larger areas (i.e. their size is subjectively determined), the shape of the occupancy distribution will be greatly affected. In other words, the shape of the occupancy distribution for one sampling unit size might be vastly different compared to the achieved results when another sample unit size was used. Furthermore, the identification of satellite and core species is not absolute but also depends on the chosen sample unit size. When designating a smaller sample unit, core species may shift to a class of medium occupancy, while when using a larger unit, satellite species may also shift to a class of intermediate occupancy. One and the same species may even be a satellite or a core species within the same spatial extent depending simply on the chosen sample unit size. Thus, the status of being a satellite species or a core species may not be an inherent feature of a species. These issues suggest that caution must be exercised when biological causes of occupancy distributions are discussed or when the core-satellite dichotomy is used as a theoretical basis for the grouping of species with differing biological characteristics. However, in cases where the sample units represent naturally delimited, discrete patches, such as clearings (Hanski 1982b), logs (Söderström 1989), or dune slacks (Bossuyt et al. 2004), these concerns are not relevant.

\section{Conclusions}

All artefactual parameters that were studied do indeed influence the number of satellite and/or core species and the shape of the occupancy distributions of the assemblages studied. Increases in sample number, sample extent, sample intensity, and sample coverage as a consequence of increasing sample number all led to a unimodal occupancy distribution with a satellite mode (Table 5). An increase in sample unit size is the only sampling protocol parameter that resulted in a core mode and a bimodal occupancy distribution. Therefore, we can deduce that a unimodal distribution with a satellite mode can be considered the 'normal' shape of the occupancy distribution in plant communities dominated by perennial, herbaceous plants. In this case, the label of 'normal' applies based on the stable existence of a satellite mode even when sampling protocol parameters are varied. The satellite mode most likely is the result of a high number of rare, low-abundance species that are present in a great majority of communities. However, the core mode seems to be a pure artefact of the sampling protocol. It occurs almost automatically when sample unit size is relatively large compared to the sample extent. Furthermore, the changes in the shape of the occu- 
Table 5. Responses of the number of satellite species, the number of core species, and the shape of the occupancy distributions to the increase of different sampling protocol parameters. Notes: ${ }^{1}$ as a result of an increase in size of sample unit, ${ }^{2}$ as a result of an increase in number of sample units, $\mathrm{H}$ = highest mode in the core class, $\mathrm{L}=$ highest mode in the satellite class.

\begin{tabular}{|c|c|c|c|c|c|}
\hline & $\begin{array}{c}\text { size of } \\
\text { sample unit }\end{array}$ & $\begin{array}{c}\text { number of } \\
\text { sample units }\end{array}$ & $\begin{array}{l}\text { Increase in } \\
\text { sample extent } \\
\text { (study area) }\end{array}$ & $\begin{array}{l}\text { Sample } \\
\text { coverage }\end{array}$ & $\begin{array}{c}\text { Sample } \\
\text { intensity }\end{array}$ \\
\hline & $\mathrm{g}$ & $\mathrm{n}$ & e & $S C=(g \times n) e^{-1}$ & $\mathrm{SI}=\mathrm{SC} \times\left(\mathrm{n} \times \mathrm{e}^{-1}\right)$ \\
\hline $\begin{array}{l}\text { Satellite } \\
\text { species }\end{array}$ & \pm constant & increase & increase & $\begin{array}{c} \pm \text { constant }^{1} \\
\text { increase }^{2}\end{array}$ & increase \\
\hline $\begin{array}{l}\text { Core } \\
\text { species }\end{array}$ & increase & \pm constant & \pm constant & $\begin{array}{c}\text { increase }^{1} \\
\pm \text { constant }^{2}\end{array}$ & decrease \\
\hline $\begin{array}{l}\text { Occupancy } \\
\text { distribution }\end{array}$ & bimodal H & unimodal L & unimodal L & $\begin{array}{l}\text { bimodal } \mathrm{H}^{1} \\
\text { unimodal } \mathrm{L}^{2}\end{array}$ & unimodal L \\
\hline
\end{tabular}

pancy distributions following changes in sampling protocol parameters can largely be explained by the movements of single species between the occupancy classes. In this context, the proportion of resolved spatial variability plays an important role. Finally, our results suggest that the impact of the sampling protocol, especially sample unit size, on the occurrence and height of modes is more relevant than the effects of other factors, such as the shape of the underlying species abundance distribution and the degree of intraspecific aggregation. Consequently, the concurrent influence of artefactual mechanisms must be excluded or separated when studying biological causes of the shape of occupancy distributions.

The similarity in the shapes of occupancy distributions from different spatial scales suggests that occupancy distribution is a self-similar pattern (Harte et al. 1999, Wilson and Chiarucci 2000, Hui and McGeoch 2007). However, whether the same artefactual mechanisms as described in this study also act on scales beyond that of local communities is yet to be determined.

Acknowledgments - Our appreciation is extended to Joel Adler and Christian Schöb for their helpful discussions and valuable comments on this manuscript. The study was supported by the following institutions: Seva-Lotteriefonds des Kantons Bern, Stiftung zur Förderung der wissenschaftlichen Forschung an der Univ. Bern, and Hochschulstiftung der Burgergemeinde Bern.

\section{References}

Aeschimann, D. and Heitz, C. 1996. Index synonymique de la flore de Suisse. - Documenta Floristicae Helvetiae 1: $1-318$.

Ashby, E. 1935. The quantitative analysis of vegetation. - Ann. Bot. 49: 779-802.
Bossuyt, B. et al. 2004. Scale-dependent frequency distributions of plant species in dune slacks: dispersal and niche limitation. - J. Veg. Sci. 15: 323-330.

Brown, J. H. 1984. On the relationship between abundance and distribution of species. - Am. Nat. 124: 255-279.

Collins, S. L. and Glenn, S. M. 1990. A hierarchical analysis of species' abundance patterns in grassland vegetation. - Am. Nat. 135: 633-648.

Collins, S. L. and Glenn, S. M. 1997. Effects of organismal and distance scaling on analysis of species distribution and abundance. - Ecol. Appl. 7: 543-551.

Curtis, J. T. and McIntosh, R. P. 1950. The interrelations of certain analytic and synthetic phytosociological characters. - Ecology 31: 434-455.

Dewdney, A. K. 1998. A general theory of the sampling process with application to the 'veil line'. - Theor. Popul. Biol. 54: 294-302.

Dewdney, A. K. 2000. A dynamical model of communities and a new species-abundance distribution. - Biol. Bull. 198: $152-165$.

Gaston, K. J. and Blackburn, T. M. 2000. Pattern and process in macroecology. - Blackwell.

Gibson, D. J. et al. 1999. The core-satellite species hypothesis provides a theoretical basis for Grime's classification of dominant, subordinate, and transient species. - J. Ecol. 87: 1064-1067.

Gibson, D. J. et al. 2005. Species frequency dynamics in an oldfield succession: effects of disturbance, fertilization and scale. - J. Veg. Sci. 16: 415-422.

Gleason, H. A. 1929. The significance of Raunkiaer's law of frequency. - Ecology 10: 406-408.

Glenn, S. M. and Collins, S. L. 1990. Patch structure in tallgrass prairies: dynamics of satellite species. - Oikos 57: 229-236.

Golicher, D. J. et al. 2006. Lifting a veil on diversity: a Bayesian approach to fitting relative-abundance models. - Ecol. Appl. 16: 202-212.

Goodall, D. W. 1952. Quantitative aspects of plant distribution. - Biol. Rev. 27: 194-245. 
Greig-Smith, P. 1964. Quantitative plant ecology. - Butterworths.

Green, J. L. and Plotkin, J. B. 2007. A statistical theory for sampling species abundances. - Ecol. Lett. 10: 1037-1045.

Guo, Q. et al. 2000. Abundance and distribution of desert annuals: are spatial and temporal patterns related? - J. Ecol. 88: 551-560.

Hanski, I. 1982a. Dynamics of regional distribution: the core and satellite species hypothesis. - Oikos 38: 210-221.

Hanski, I. 1982b. Distributional ecology of anthropochorous plants in villages surrounded by forest. - Ann. Bot. Fenn. 19: $1-15$.

Hanski, I. et al. 1993. Three explanations of the positive relationship between distribution and abundance of species. - In: Ricklefs, R. E. and Schluter, D. (eds), Species diversity in ecological communities. Univ. of Chicago Press, pp. 108166.

Harte, J. et al. 1999. Self-similarity in the distribution and abundance of species. - Science 284: 334-336.

Hartley, S. 1998. A positive relationship between local and regional occupancy is almost inevitable (but not all positive relationships are the same). - J. Anim. Ecol. 67: 992-994.

Heatherly, T. et al. 2007. Stream insect occupancy-frequency patterns and meta-population structure. - Oecologia 151: 313-321.

Heino, J. 2005. Positive relationship between regional distribution and local abundance in stream insects: a consequence of niche breadth or niche position? - Ecography 28: 345354.

Heino, J. and Virtanen, R. 2006. Relationships between distribution and abundance vary with spatial scale and ecological group in stream bryophytes. - Freshwater Biol. 51: 18791889.

Hughes, R. G. 1986. Theories and models of species abundance. - Am. Nat. 128: 879-899.

Hui, C. and McGeoch, M. A. 2007. A self-similarity model for the occupancy frequency distribution. - Theor. Popul. Biol. 71: 61-70.

Keeley, J. E. et al. 2005. Factors affecting plant diversity during post-fire recovery and succession of Mediterranean-climate shrublands in California, USA. - Div. Distr. 11: 525-537.

Laube, S. and Zotz, G. 2007. A meta-population approach to the analysis of long-term changes in the epiphyte vegetation on the host tree Annona glabra. - J. Veg. Sci. 18: 613-624.

Löbel, S. et al. 2006. Meta-population processes in epiphytes inferred from patterns of regional distribution and local abundance in fragmented forest landscapes. - J. Ecol. 94: $856-868$.

Lobo, J. M. and Favila, M. E. 1999. Different ways of constructing octaves and their consequences on the prevalence of the bimodal species abundance distribution. - Oikos 87: 321-326.

Magurran, A. E. and Henderson, P. A. 2003. Explaining the excess of rare species in natural species abundance distributions. - Nature 422: 714-716.

May, R. M. 1975. Patterns of species abundance and diversity. - In: Cody, M. L. and Diamond, J. M. (eds), Ecology and evolution of communities. Belknap Press, pp. 81-120.

McGeoch, M. A. and Gaston, K. J. 2002. Occupancy frequency distributions: patterns, artefacts and mechanisms. - Biol. Rev. 77: 311-331.
McGill, B. J. et al. 2007. Species abundance distributions: moving beyond single prediction theories to integration within an ecological framework. - Ecol. Lett. 10: 995-1015.

McIntosh, R. P. 1962. Raunkiaer's 'law of frequency'. - Ecology 43: 533-535.

Mueller-Dombois, D. and Ellenberg, H. 1974. Aims and methods of vegetation ecology. - Wiley.

Nee, S. et al. 1991. Core and satellite species: theory and artefacts. - Oikos 62: 83-87.

Papp, L. and Izsak, J. 1997. Bimodality in occurrence classes: a direct consequence of lognormal or logarithmic series distribution of abundances - a numerical experimentation. Oikos 79: 191-194.

Parmesan, C. 2006. Ecological and evolutionary responses to recent climate change. - Annu. Rev. Ecol. Evol. Syst. 37: 637-669.

Pärtel, M. et al. 2001. Variation in species richness within and between calcareous (alvar) grassland stands: the role of core and satellite species. - Plant Ecol. 157: 203-211.

Perelman, S. B. et al. 2001. Cross-scale vegetation patterns of flooding pampa grasslands. - J. Ecol. 89: 562-577.

Pielou, E. C. 1975. Ecological diversity. - Wiley.

Preston, F. W. 1948. The commonness, and rarity, of species. Ecology 29: 254-283.

Raunkiaer, C. 1934. Statistical researches on plant formations. In: Raunkiaer, C. (ed.), The life forms of plants and statistical plant geography. Clarendon Press, pp. 379-424.

Scheiner, S. M. and Rey-Benayas, J. M. 1997. Placing empirical limits on meta-population models for terrestrial plants. - Evol. Ecol. 11: 275-288.

Söderström, L. 1989. Regional distribution patterns of bryophyte species on spruce logs in northern Sweden. - Bryologist 92: 349-355.

Soininen, J. and Heino, J. 2005. Relationships between local population persistence, local abundance, and regional occupancy of species: distribution patterns of diatoms in boreal streams. - J. Biogeogr. 32: 1971-1978.

Storch, D. and Sizling, A. L. 2002. Patterns of commonness and rarity in central European birds: reliability of the coresatellite hypothesis within a large scale. - Ecography 25: 405-416.

Tokeshi, M. 1992. Dynamics of distribution in animal communities: theory and analysis. - Res. Popul. Ecol. 34: 249273.

Ulrich, W. and Zalewski, M. 2006. Abundance and co-occurrence patterns of core and satellite species of ground beetles on small lake islands. - Oikos 114: 338-348.

Van Rensburg, B. J. et al. 2000. Testing generalities in the shape of patch occupancy frequency distributions. - Ecology 81: 3163-3177.

Walther, G. R. et al. 2002. Ecological responses to recent climate change. - Nature 416: 389-395.

Whittaker, R. H. 1965. Dominance and diversity in land plant communities. - Science 147: 250-260.

Wiens, J. A. 1989. Spatial scaling in ecology. - Funct. Ecol. 3: 385-397.

Williams, C. B. 1950. The application of the logarithmic series to the frequency of occurrence of plant species in quadrats. - J. Ecol. 38: 107-138.

Williams, C. B. 1964. Patterns in the balance of nature.-Academic Press. 
Wilson, J. B. and Chiarucci, A. 2000. Do plant communities exist? Evidence from scaling-up local species-area relations to the regional level. - J. Veg. Sci. 11: 773-775.
Wright, D. H. 1991. Correlations between incidence and abundance are expected by chance. - J. Biogeogr. 18: 463-466. 